\title{
Anaerobic degradation of polyethylene glycol 400
}

\author{
Xia Chen ${ }^{1,2, a}$, Yangdong $\mathrm{Hu}^{1, \mathrm{~b}}$ \\ ${ }^{1}$ College of Chemistry and Chemical Engineering,Ocean University of China, Qingdao 266100 , \\ Shandong Province, China; \\ ${ }^{2}$ Department of Environmental Science and Engineering, Qilu University of Technology ,Jinan, \\ 250353, Shandong Province, China \\ ajudych@163.com; bydhuhd@ouc.edu.cn
}

Keywords: anaerobic sludge, polyethylene glycol 400(PEG400), biodegradation

Abstract: Anaerobic degradation of the polyethylene glycol 400(PEG400) was studied. The effect of concentration was respectively assessed on the anaerobic bio-degradation of PEG400, using the organic model wastewater containing the $1.0 \%$ and $0.5 \%$ PEG400. The result showed that: the TOC(Total Organic Carbon) and COD(Chemical Oxygen Demand) removal rate of the 1.0\% PEG400 was degraded more than 50\% in 5-day HRT(hydraulic retention time) and by $90 \%$ in 10-day HRT(hydraulic retention time), and the total gas yield was more than $1000 \mathrm{ml}$. The reaction process was in accordance with the anaerobic reaction kinetics. It achieved ideal treatment effection by anaerobic treatment of high concentration organic wastewater.

\section{Introduction}

In the recent years, the amounts of the waste water and pollutants were discharged to the environment duing to the development of the industry and social economy. Waste water often contain highly concentrated organic matters which discharged from pulp and paper mill, food, chemical synthetic pharmacy, leather, chemical and industries . The character of the waste water is highly concentrated organic matters and is hard to be biodegraded. Polyethylene glycol (PEG), an organic water soluble polymer whose structure is $\mathrm{H}\left(\mathrm{OCH}_{2} \mathrm{CH}_{2}\right) n \mathrm{OH}$, is used in a wide range of applications, such as in the production of water-soluble lubricants, cosmetics, surfactants, antifreeze agents, photo-voltaic, plastics and pharmaceutical preparations ${ }^{[1]}$. After industrial utilization, a high proportion of them enter conventional waste-water treatment plants (WWTPs) ${ }^{[2]}$.

Over the last decade, the treatment of organic containing waste-waters by means of integrated chemical-biological processes has received a lot of attention ${ }^{[3]}$. Both chemical and biological processes of waste-water treatment have inherent limitations in applicability, effectiveness and cost. The bio-degradation of PEG under anaerobic or aerobic conditions depends on the molecular weight(MW) and the structural conformation of PEG molecules ${ }^{[3]}$. Suzuki et al. ${ }^{[4,5]}$ reported that a significant improvement in biodegradability occurred after ozonation pretreatment and the molecular weight of 300 or lower could be biodegradable. Otal E. et al. ${ }^{[6]}$ studied that various polyethylene glycols in a molecular weight ranged from 200 to 35000 and it was found that biodegradability generally decreased with increasing molecular weight. An overall $80 \%$ TOC removal could be achieved when WAO was coupled with a 0.5 -day residence time of subsequent biological treatment, while only about 60-70\% TOC removal could be achieved after a 4-day residence time of direct biological treatment. In order to improve treatment of the PEG-containing wastewaters and to foresee risks associated with a possible accidental PEG releasing, the biodegradability of PEG 400 was investigated in this study. In particular, the degradative capacities of diverse concentration of 
the PEG400 was examined. It was found that biodegradability generally decreased with increasing MW. A synergistic, mixed culture of Flavobacterium and Pseudomonas species was able to utilize PEGs of up to 20,000 Da MW and tetraethylene glycol (TEG, MW 194) was aerobic degraded leading to TEG-mono- and dicarboxylic acids, tri-, di andmonoethylene glycol as metabolites, analyzed by gas chro-matography mass spectrometry (GC-MS) ${ }^{[7]}$.Microbes isolated from the sludge of a WWTP efficiently decomposed PEG up to 20,000 Da in size, either under aerobic or anaerobic conditions ${ }^{[2]}$. Anaerobic degradation of PEGs of up to 20,000 Da MW using freshwater media has been reported with bacteria isolated from anaerobic reactors of urban WWTPs ${ }^{[8,9]}$.

\section{Materials and methods}

\section{Acclimation of activated sludge}

The initial inoculum for the experiment consisted of digested sludge obtained from an urban wastewater treatment plant. The sludge was digested in a water bath with the temperature of $38^{\circ} \mathrm{C}$ after some time until the digested sludge could adapt to the anaerobic environment of the PEG .The $1000 \mathrm{ml}$ reactors were placed in the water bath, which included $400 \mathrm{ml}$ digested sludge and $1000 \mathrm{ml}$ simulate wastewater of PEG400.

\section{Materials and apparatus}

Commercial PEG400 with the molecular weights(MW) was provided and used without further purification. The main experimental materials and reagents were shown in table1.

Table1 Experimental materials and reagents

\begin{tabular}{ccl}
\hline Name & Grade & \multicolumn{1}{c}{ Manufacture factory } \\
\hline PEG400 & $\mathrm{AR}$ & Big Damaol reagent factory in Tianjin \\
$\mathrm{H}_{2} \mathrm{SO}_{4}$ & $\mathrm{AR}$ & Guangzhou chemical reagent factory \\
$\mathrm{NaOH}$ & $\mathrm{AR}$ & The chemical reagent factory in Tianjin \\
$\mathrm{PEG} 2000$ & $\mathrm{AR}$ & Big Damaol reagent factory in Tianjin \\
$30 \mathrm{HH}_{2} \mathrm{O}_{2}$ & $\mathrm{AR}$ & Guangzhou chemical reagent factory \\
$\left(\mathrm{NH}_{4}\right)_{2} \mathrm{SO}_{4} \cdot \mathrm{FeSO}_{4} \cdot 6 \mathrm{H}_{2} \mathrm{O}$ & $\mathrm{AR}$ & The chemical reagent factory in Tianjin \\
$\mathrm{TOC}^{\text {Analyzer }}$ & & Shi-madzu co., LTD \\
Water bath & & Jiangsu Jintan Ronghua instrument co., LTD \\
Electronic scales & & Shanghai precision scientific instrument co., LTD \\
$\mathrm{COD}$ Analyzer & & Qingdao Jiade Li technology co., LTD \\
pH Meter & & Qingdao Renhe Xing experiment technology co., \\
& & LTD
\end{tabular}

\section{Analytical methods}

The $\mathrm{pH}$ was measured by $\mathrm{pH}$ Meter and $\mathrm{COD}_{\mathrm{cr}}$ was measured by the dichromate method ${ }^{[8]}$, TOC was measured with a Shi-madzu 5050 TOC Analyzer whose operation was based on combustion/non-dispersive infrared (NDIR) gas analysis. The gas yield was measured by drainage method.

\section{Experiment procedure}

In the experiment, we used the anaerobic sludge to degrad the PEG400, and the biomass capable of degrading PEG with using PEG400 as only carbon and energy source ${ }^{[9]}$. The concentration of PEG400 simulate wastewater was $1.0 \% 、 0.5 \%$. The initial TOC and $\mathrm{COD}_{\mathrm{cr}}$ respectively was $5426 \mathrm{mg} / \mathrm{L} 、 2932 \mathrm{mg} / \mathrm{L}$ and $16800 \mathrm{mg} / \mathrm{L} 、 9600 \mathrm{mg} / \mathrm{L}$. Degradation of PEGs were determined by analyzing the aqueous COD and TOC. 


\section{Experimental results and discussion}

\section{The TOC and COD decreased with the change of time}

The concentration of PEG400 respectively was $1.0 \%$ and $0.5 \%$, and the $\mathrm{TOC}_{0}$ was $5426 \mathrm{mg} / \mathrm{L}$ 、 $2932 \mathrm{mg} / \mathrm{L}$. The fig. 1 showed the regular transformation of the TOC and the TOC/TOC 0 following the change of time. The different concentration of PEG400 were biodegradable under the experimental conditions and the PEG400 was used as the soul energy and carbon source ${ }^{[9,10]}$. The $\mathrm{TOC} \mathrm{TOC}_{0}$ of $1.0 \%$ PEG400 was degraded more than $50 \%$ in 5-day and by $90 \%$ in 10-day, but the $0.5 \%$ PEG400 was degraded slowly, it was less than $20 \%$ in 4 days. Then the $0.5 \%$ PEG400 was degraded rapidly after 4 days and more than $80 \%$ in 10 days. In the fig. 1 , the TOC reduced with the change of time, the $1.0 \%$ PEG400 TOC was degraded slowly in the first time, while the lag time increased. The result showed that the TOC removal was reached the ideal effect. The data of the Fig2. was the removal rate of $\mathrm{COD}$ and the $\mathrm{COD}_{\mathrm{cr}}$ decreased following the change of time. The $\mathrm{COD}_{\mathrm{cr}}$ removal rate was degraded more than $60 \%$ in 5 -day and by $90 \%$ in 10-day, which was similar to the TOC removal rate. The Fig1. and Fig2. showed that the model wastewater of PEG400 could be biodegradable in anaerobic oxidation.

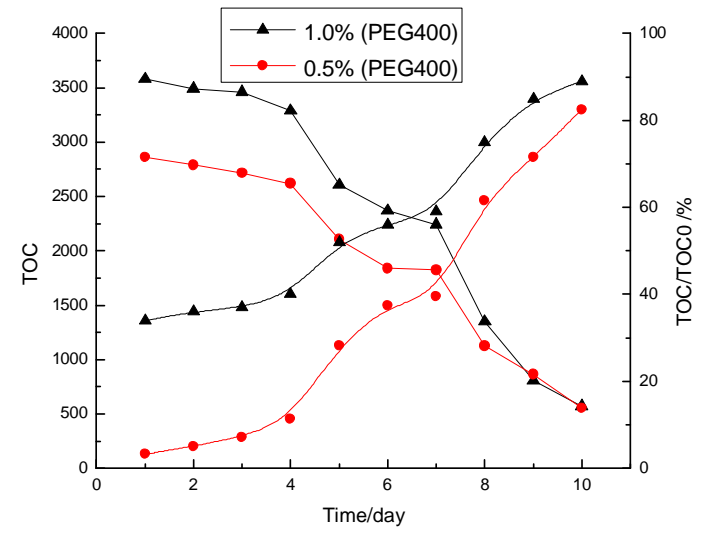

Fig. 1. TOC and TOC/TOC ${ }^{0}$ decreased during continuous anaerobic oxidation

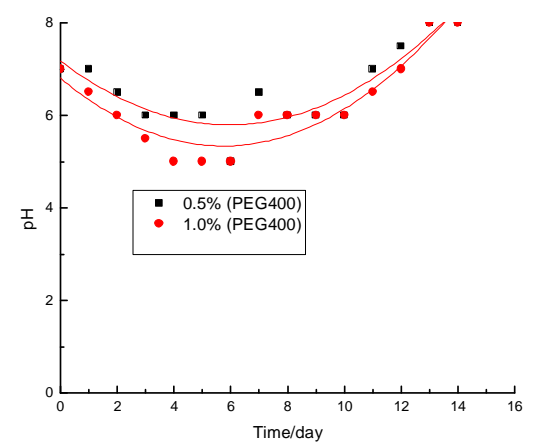

Fig. 3. pH changed during continuous anaerobic oxidation

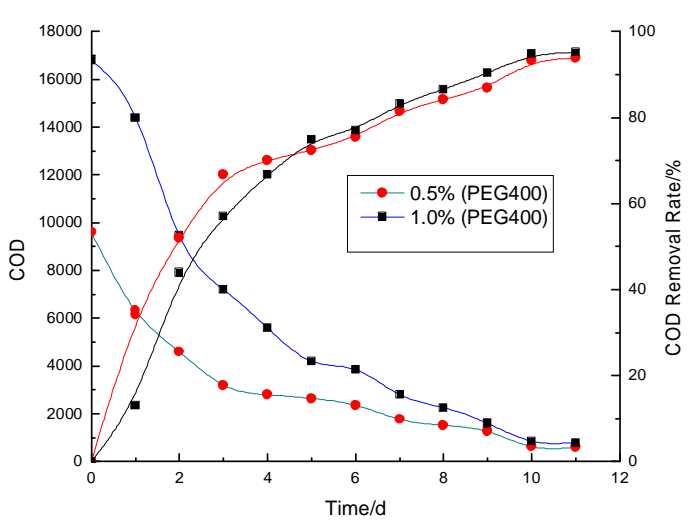

Fig.2 COD decreased during continuous anaerobic oxidation

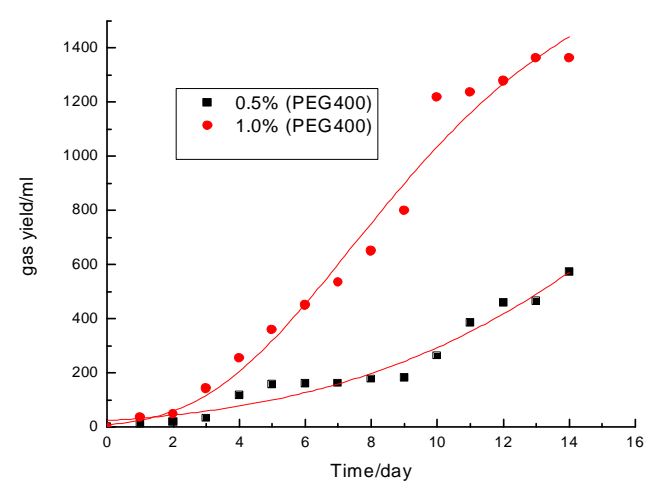

Fig. 4. Gas yield during continuous anaerobic oxidation 


\section{The change of $\mathrm{pH}$ and the gas production}

The result presented in Fig3. indicated that the $\mathrm{pH}$ changed during anaerobic oxidation with the polynomial fit of the $0.5 \%$ PEG400 and $1.0 \%$ PEG400. The result of polynomial fit was accord with the anaerobic reaction mechanism: the $\mathrm{pH}$ of model wastewater changed from neutral gradually declining and then rising. The Fig3. showed that the $\mathrm{pH}$ of the 1.0\% PEG400 was slightly lower than $0.5 \%$ PEG400, the $\mathrm{pH}$ was lowest in the forth day and then raised. The Fig4. was the gas production in the anaerobic oxidation which was gradually rising with the reaction time. The gas production was accord with the anaerobic reaction mechanism.

\section{Conclusions}

The experimental results indicate that the efficiency of degradation in terms of the time required to reach the ideal effect. The critical hydraulic retention times was a key factor, which was estimated of the experiment. The removal efficiency of COD and TOC was more than $90 \%$ in 10-day. The removal rate of $0.5 \%$ PEG400 was better than $1.0 \%$ PEG400 in five days ago, and then the removal of $1.0 \%$ PEG400 was higher than the $0.5 \%$ PEG400. The $\mathrm{pH}$ and gas production was accord with the anaerobic reaction mechanism. The result showed that the maximum growth efficiency of the anaerobic culture using PEG as the only carbon and energy source wasn't affected by the concentration of PEG400

\section{Acknowledgements}

Authors thank all the students and colleagues responsible for the friendly atmosphere at the experiment and to all institutions supporting research.

\section{Reference}

[1] Emilia Otal, Julian Lebrato.Anaerobic degradation of polyethylene glycol mixtures.Chem Technol Biotechnol. 2003;78:1075-1081

[2]Huang, Y.-L., Li, Q.-B., Deng, X., Lu, Y.-H., Liao, X.-K., Hong, M.-Y.,Wang, Y., . Aerobic and anaerobic biodegradation of polyethylene glycols using sludge microbes. Process Biochem.2005;40:207-211.

[3] White G.F., Russel N.J. and Tidswell E.C., Bacterial scission of ether bonds. Microbiol. Rya., 60. 216-232 (1996).

[4] Suzuki J, Nakagawa H, Ito H. Study on ozone treatment of water-soluble polymers, II, Utilization of ozonized polyethylene glycol by bacteria. J Appl Polym Sci 1976;20:2791-9.

[5] Suzuki J, Hukushima K, Suzuki S. Effect of ozone treatment upon biodegradability of water-soluble polymers. Environ Sci Technol 1978;12:1180-3.

[6] Otal E., Mantzavinos D., Delgado M.V., Hellenbrand R, Lebrato J., Metcalfe IS. and Livingston A.G., Integrated wet air oxidation and biological treatment of polyethylene glycol-containing wastewaters. J.Chem.Technol. Biotedtnol, 70,147-156(1997)

[7] Kawai, F., Kimura, T., Fukaya, M., Tani, Y., Ogata, K., Ueno, T.,Fukami, H., 1978. Bacterial oxidation of polyethylene glycol.Appl. Environ. Microbiol. 35 (4), 679-684.

[8]Dwyer, D.F., Tiedje, J.M., 1983. Degradation of ethylene glycol andpolyethylene glycols by methanogenic consortia. Appl.Environ. Microbiol. 46 (1), 185-190.

[9]Otal, E., Lebrato, J. Anaerobic degradation of polyethylene glycol mixtures. J. Chem. Technol. Biotechnol., 2003,78, 1075-1081. 